\title{
LA EVALUACIÓN AMBIENTAL ESTRATÉGICA (EAE) COMO INSTRUMENTO DE VALORACIÓN ESPACIAL. ANÁLISIS DE SU IMPLEMENTACIÓN EN PROYECTOS DE INFRAESTRUCTURA VIAL
}

\section{THE STRATEGIC ENVIRONMENTAL ASSESSMENT (EAE) AS A SPACE ASSESSMENT INSTRUMENT. ANALYSIS OF ITS IMPLEMENTATION IN ROAD INFRASTRUCTURE PROJECTS}

\author{
Prof. Sandra Milena Carabuena Mendoza \\ Bióloga \\ Maestrante de Gestión Socioambiental - I Cohorte \\ Universidad de Ciencias Aplicadas y Ambientales (UDCA) \\ http://dx.doi.org/10.30972/geo.14282746
}

RESUMEN

El presente artículo tiene por fundamento, esbozar las ideas centrales que subyacen en la denominada Evaluación Ambiental Estratégica (EAE). Este instrumento de evaluación ambiental, relativamente nuevo para muchos sectores entre ellos el de la infraestructura vial, ha nacido en Europa y en la actualidad, su implementación representa un desafío en los países de América Latina.

Con base en los intereses mencionados, el objetivo del artículo consiste en analizar la implementación de la EAE en proyectos de infraestructura vial, a fin de brindar los lineamentos y recomendaciones para su aplicación en proyectos que puedan generar algún tipo de afectación a nivel ambiental.

Los pasos metodológicos han consistido en la selección, revisión y análisis de material bibliográfico referido a la temática y la comparación de casos significativos de implementación de la EAE en países iberoamericanos. Los resultados obtenidos generarán un insumo para el diseño de líneas acción cuyo fin será la implementación en la planificación territorial.

\begin{abstract}
The present article is based on outlining the central ideas underlying the so-called Strategic Environmental Assessment (SEA). This instrument of environmental evaluation, relatively new for many sectors, including road infrastructure, was born in Europe and today, its implementation represents a challenge in the countries of Latin America.

Based on the mentioned interests, the objective of the article is to analyze the implementation of SEA in road infrastructure projects, in order to provide guidelines and recommendations for their application in projects that may generate some type of environmental impact.

The methodological steps have consistedof the selection, review and analysis ofbibliographic material referred to thesubject matter and the comparison of significant cases of implementation of EAEin Ibero-American countries. The resultswill generate an input for the design oflines action whose purpose is theimplementation planning.
\end{abstract}

\section{PALABRAS CLAVES}

Evaluación Ambiental Estratégicas; Instrumento; Infraestructura vial; lineamientos; recomendaciones; planificación territorial.

\section{KEY WORDS}

Strategic environmental assessment, Road infrastructure, land planning, guidelines, recommendation Publicado en formato digital: Prof. Sandra Milena Carabuena Mendoza. LA EVALUACION AMBIENTAL ESTRATÉGICA (EAE) COMO INSTRUMENTO DE VALORACIÓN ESPACIAL. ANÁLISIS DE SU IMPLEMENTACIÓN EN PROYECTOS DE INFRAESTRUCTURA VIAL. Revista Geográfica Digital. IGUNNE. Facultad de Humanidades. UNNE. Año 14. № 28. Julio Diciembre 2017. ISSN 1668-5180. Resistencia, Chaco. En: http://hum.unne.edu.ar/revistas/geoweb/default.htm - http:// dx.doi.org/10.30972/geo.14282746 
Revista Geográfica Digital. IGUNNE. Facultad de Humanidades. UNNE. Año 14. № 28.

Julio - Diciembre 2017. ISSN 1668-5180 Resistencia, Chaco

\section{INTRODUCCIÓN}

El presente artículo, tiene como finalidad establecer las pautas para desarrollar los lineamientos y recomendaciones para proyectos de infraestructura vial en el marco de la Evaluación Ambiental Estratégica,

La Evaluación Ambiental Estratégica, "es entendida como un proceso sistemático que incorpora las consideraciones y criterios ambientales en los momentos claves del proceso de planeación de decisiones estratégicas, garantizando el equilibrio entre la sostenibilidad ambiental y los planes de desarrollo socioeconómico de un país, un sector y/o región". (Conferencia Europea de Ministros de Transporte - ECMT 1998). Este concepto, permitió su incorporación a los objetivos del Plan Nacional de Desarrollo 2002-2006 "Hacia un Estado Comunitario"; el cual identificó que uno de los aspectos principales del programa de Sostenibilidad Ambiental de la Producción Nacional, debería ser el impulso a la incorporación de la dimensión ambiental en los procesos de planificación sectorial, con énfasis en las Evaluaciones Ambientales Estratégicas, (EAE), y el fortalecimiento del actual proceso de licenciamiento ambiental.

Es así que, el Ministerio de Ambiente, Vivienda y Desarrollo Territorial (MAVDT) de Colombia, en cabeza de la Dirección de Desarrollo Sectorial Sostenible, vienen promoviendo desde el 2002 la incorporación de las evaluaciones ambientales estratégicas en la formulación de los diferentes planes sectoriales del país, tema a profundizar en el presente documento; haciendo énfasis en dos estudios de caso: la conexión vial Orinoquia - Pacifico (Departamentos de Valle del Cauca - Tolima - Huila) y la Vía Bogotá - Villavicencio (Departamentos Cundinamarca - Meta). De tal modo que este documento se constituye en la base del marco conceptual, referencial y metodológico de futuras EAEs en el sector de la infraestructura vial, es decir, como material de referencia para incorporar el procedimiento de EAE en las diferentes políticas, planes y programas que tengan relación con el tema. Del mismo modo expone los lineamientos generales para la aplicación de las diferentes fases de la metodología de la EAE, en proyectos de infraestructura vial.

Por otro lado se hace preciso mencionar que la mayoría de los profesionales que han trabajo en el tema, ven a la EAE como una ayuda a la decisión en lugar de un proceso de toma de decisiones (como un EIA), es decir se considera una herramienta para la planeación anticipada para ser flexiblemente aplicada en varios escenarios del ciclo de creación de políticas. Bajo esta perspectiva amplia la EAE engloba evaluaciones de iniciativas de políticas amplias y planes y programas más concretos que tienen referencias físicas y espaciales (e.g. planes regionales y de ciudades, programas de desarrollo regional). Con este alcance de la cobertura, un problema se convierte en evidente. Las metodologías para ser aplicadas en los extremos opuestos del espectro de toma de decisiones, difieren marcadamente. Sin embargo, los principios del EIA se aplican en todos los niveles (Dalal-Clayton \& Sadler, 1999).

Para cumplir con lo anteriormente descrito, se ha realizado una revisión del estado del arte del tema, interpretando la metodología de una EAE con base en las diferentes aplicaciones que hasta la fecha se han realizado en el ámbito internacional y nacional. En el desarrollo de este documento se adoptó como base la metodología propuesta por el Departamento Nacional de Planeación, debido a que ésta recoge los aspectos más relevantes de las experiencias internacionales, acondicionando el modelo conceptual y procedimental a la situación nacional.

Para el presente documento es importante establecer la diferencia entre los dos tipos de evaluación ambiental la EIA (Evaluación de Impacto Ambiental) y la EAE (Evaluación Ambiental Estratégica), cabe mencionar que la EIA y EAE son dos instrumentos integrales de un Sistema de Evaluación Ambiental y como tal persiguen el objetivo común de controlar y prevenir los impactos negativos al medio ambiente. La EIA se sustenta en la capacidad de anticipación de las consecuencias que se puedan presentar por el desarrollo de actividades, obras y proyectos en el entorno, mientras que la

Publicado en formato digital: Prof Sandra Milena Carabuena Mendoza. LA EVALUACION AMBIENTAL ESTRATÉGICA COMO INSTRUMENTO DE VALORACIÓN ESPACIAL. ANÁLISIS DE SU IMPLEMENTACIÓN EN PROYECTOS DE INFRAESTRUCTURA VIAL. Revista Geográfica Digital. IGUNNE. Facultad de Humanidades. UNNE. Año 14. № 28. Julio Diciembre 2017. ISSN 1668-5180. Resistencia, Chaco. En: http://hum.unne.edu.ar/revistas/geoweb/default.htm 
Revista Geográfica Digital. IGUNNE. Facultad de Humanidades. UNNE. Año 14. № 28.

Julio - Diciembre 2017. ISSN 1668-5180 Resistencia, Chaco

EAE representa un nivel más estratégico, de planificación toda vez que su integración se puede realizar en un plan, política o programa.

\section{MARCO TEÓRICO}

La Evaluación Ambiental Estratégica (EAE) es un ajuste al proceso analítico y participativo para la incorporación de consideraciones ambientales durante la toma de decisiones en las etapas iniciales, dentro de las políticas, planes y programas que afectan los recursos naturales. La EAE evalúa en un alto nivel estratégico, los impactos ambientales de un proyecto en el contexto de los factores sociales y económicos. Esto excede la tradicional Evaluación de Impactos Ambientales (EIA), la cual se enfoca en el impacto ambiental biótico, abiótico y sociocultural. La EAE promueve y provee consideraciones críticas sistemáticas a nivel sectorial, regional y nacional para suscitar la sostenibilidad ambiental, el crecimiento inteligente y la prevención de la contaminación. El término "EAE" involucra un espectro de procesos analíticos tales como La Evaluación Ambiental y Social Estratégica (EASE), la Evaluación Socioambiental Estratégica (ESE), el Análisis Ambiental del País $(A A P)$, la Evaluación Combinada Estratégica del Impacto (ECEI) y la Evaluación Acumulativa del Impacto (EAI) Guidance Notes on Tools for Pollution Management. A continuación, se presentan las diferencias entre la EAE y EIA:

\begin{tabular}{|c|c|}
\hline Evaluación Impacto Ambiental & Evaluación Ambiental Estratégica \\
\hline Se aplica a actividades, obras o proyectos & Políticas, planes y programas \\
\hline $\begin{array}{l}\text { Es solicitada por el titular, propietario o } \\
\text { representante legal de una actividad, obra o } \\
\text { proyecto }\end{array}$ & Es requerida por la administración pública \\
\hline $\begin{array}{c}\text { Determina los impactos en el área de } \\
\text { influencia del proyecto (Área de EAE, } \\
\text { Dirección General de Cambio Climático y } \\
\text { Asuntos Estratégicos) }\end{array}$ & $\begin{array}{l}\text { Permite visualizar los impactos macro } \\
\text { acumulativos }\end{array}$ \\
\hline $\begin{array}{l}\text { Permite definir planes de manejo para mitigar, } \\
\text { controlar o compensar }\end{array}$ & $\begin{array}{c}\text { Permite evidenciar las oportunidades, riesgos, } \\
\text { amenazas, potencialidades, basadas en la } \\
\text { sustentabilidad }\end{array}$ \\
\hline & Permite tomar decisiones estratégicas \\
\hline
\end{tabular}
COMO INSTRUMENTO DE VALORACIÓN ESPACIAL. ANÁLISIS DE SU IMPLEMENTACIÓN EN PROYECTOS DE INFRAESTRUCTURA VIAL. Revista Geográfica Digital. IGUNNE. Facultad de Humanidades. UNNE. Año 14. № 28. Julio Diciembre 2017. ISSN 1668-5180. Resistencia, Chaco. En: http://hum.unne.edu.ar/revistas/geoweb/default.htm 
Revista Geográfica Digital. IGUNNE. Facultad de Humanidades. UNNE. Año 14. № 28.

Julio - Diciembre 2017. ISSN 1668-5180 Resistencia, Chaco

De acuerdo con Clayton and Sadler (1999), la práctica de la EIA es cohibida por ciertas limitaciones y debilidades. Estas incluyen debilidades estructurales centradas en el escenario tardío relativo en el cual la EIA se aplica usualmente en la toma de decisiones. En este punto, se han decidido las preguntas de alto orden sobre si, donde y que tipo de desarrollo debe llevarse a cabo, algunas veces con poco o ningún análisis ambiental. Proyecto por proyecto la EIA es también una manera inefectiva de examinar estos problemas. La EAE o un enfoque equivalente, puede ser usado como un complemento del EIA a nivel de proyecto para incorporar consideraciones ambientales y alternativas directamente dentro del diseño de las políticas, planes y programas. Así, cuando se aplica sistemáticamente "aguas arriba" del ciclo de decisión y en las políticas de comercio, fiscales y económicas que guían el curso general del desarrollo, la EAE puede ser un vector para un enfoque de sostenibilidad para la toma de decisión y planeación como fue llamado por la Comisión Brundtland (WCED 1987) y por la Agenda 21 (UNCED 1992). Este enfoque "aguas arriba" pueden también ayudar a enfocar y racionalizar las EIA de los proyectos, haciéndolos más consecuentes y reduciendo el tiempo y el esfuerzo involucrados en su elaboración. La EAE puede generar otros beneficios significativos; por ejemplo, al descartar ciertos tipos de desarrollo a nivel de política, reduciendo la necesidad de muchas EIA a nivel de proyecto y así aliviando la presión cuando la capacidad institucional y/o de habilidades es limitada.

Con base en lo anterior, y dada la escasez de experiencias de implementación de la $\mathrm{EAE}$, se evidencia la necesidad de contar con lineamientos y recomendaciones que guíen la aplicación de la EAE en proyectos de infraestructura vial. La falta de experiencias previas suficientes en materia de evaluación ambiental estratégica puede llevar a la extensión de aproximaciones inadecuadas a los modelos tradicionales de evaluación de impactos, con las consecuencias y dificultades básicas mencionadas.

En muchos contextos la EAE es un procedimiento administrativo, por ejemplo en Europa es una norma reglada por la directiva Europea, de igual forma es importante mencionar las numerosas organizaciones que reconocieron la necesidad de la EAE y están estableciendo procedimientos y lineamientos $\mathrm{O}$ investigando las posibilidades de hacerlo, es así como surgieron iniciativas importantes en la comisión europea, el banco mundial, el EBRD (European Bank of Research and Development) y la OCDE, entre otros.

La EAE es particularmente útil en el análisis ambiental y la evaluación de acciones estratégicas en el sector del transporte cuando se adopta un enfoque intermodal. Análisis recientes de la aplicación de la EAE en el sector del transporte en la UE, los Estados Unidos y Canadá demuestran que la EAE se lleva a cabo en la mayoría de estos países, tanto de forma voluntaria como obligatoria. ECMT (1998).

La aparición de las EAE tiene antecedentes a nivel internacional hacia los años ochenta, a través de las orientaciones que el Banco Mundial (BM) incluía para el desarrollo de Evaluaciones Ambientales Sectoriales (EAS) y Evaluaciones Ambientales Regionales (EAR). Actualmente, el movimiento de mayor relevancia es el iniciado por la Unión Europea, a raíz del protocolo de la Convención de Evaluación de Impacto Ambiental en el Contexto Transfronterizo, y que determinó lo necesidad de generar una reglamentación específica sobre la aplicación de la EAE, vigente a partir del 2004 (DNP, 2004).

Varias son las iniciativas y las acciones concretas de EAE que a nivel internacional se han desarrollado, entre otras las siguientes: i) Plan hidrológico Nacional de España; ii) Plan Comprensivo de Ecoturismo para Isabela - Galápagos (PCE) y su EAE (España); iii) EAE para saneamiento básico y medio ambiente (Argentina); iv) La EAE un Instrumento de Gestión Ambiental para ser Aplicado en el País (Chile); v) Metodologías paramétricas para la EAE con énfasis en índices socio ecológicos y planeamiento territorial (España); vi) La Convención de Ramsar y la evaluación de impacto: la EAE un instrumento para la revisión y rediseño de los marcos jurídicos e institucionales y para la creación de los incentivos adecuados en Suiza; vii) EAE del Plan Comprensivo de Ecoturismo para Isabela. Galápagos (Ecuador); vi ii) Taller "Evaluación Ambiental Estratégico del Sector Pesquero Argentino", y ix) EAE para un Proyecto vial en Bolivia: corredor norte de Bolivia, la Paz-Guayaramerín-Cobija. COMO INSTRUMENTO DE VALORACIÓN ESPACIAL. ANÁLISIS DE SU IMPLEMENTACIÓN EN PROYECTOS DE INFRAESTRUCTURA VIAL. Revista Geográfica Digital. IGUNNE. Facultad de Humanidades. UNNE. Año 14. № 28. Julio Diciembre 2017. ISSN 1668-5180. Resistencia, Chaco. En: http://hum.unne.edu.ar/revistas/geoweb/default.htm 
Revista Geográfica Digital. IGUNNE. Facultad de Humanidades. UNNE. Año 14. № 28.

Julio - Diciembre 2017. ISSN 1668-5180 Resistencia, Chaco

Son diversos, entonces, los sectores del desarrollo al igual que los componentes ambientales a los cuales vienen siendo aplicadas las EAE, pasando por proyectos, planes, y decisiones de política; también se han desarrollado guías a nivel mundial sobre EAE, entre las que se destacan las realizadas en Centroamérica, Canadá, Sudáfrica y Chile (DNP, 2004. Op. cit.).

\section{Situación de la implementación de la EAE en Colombia}

En Colombia la aplicación EAE es limitada, dado que tradicionalmente la evaluación ambiental de planes, programas y proyectos particularmente estos últimos se ha circunscrito al cumplimiento de los estudios (Ministerio del Medio Ambiente, 1997) exigidos por la normatividad, es decir: Diagnóstico Ambiental de Alternativas (DAA), Estudio de Impacto Ambiental (EIA) y Plan de Manejo Ambiental (PMA). A partir del análisis de los casos nacionales, es posible afirmar (DNP 2004, op. cit.) que la orientación de estos estudios no corresponde en su totalidad al enfoque de la EAE, por cuanto aquella incluye un componente importante de participación, así como la relación entre la evolución y la decisión final adoptada.

En cuanto a las iniciativas de EAE, ya sea desde el enfoque sectorial, regional o de grupos de proyectos, se inicia a mediados de los años 90 con estudios como:

1) La evaluación de posibles ubicaciones de puertos en el Pacífico y el Mar Caribe

2) El desarrollo de una estrategia de manejo de residuos peligrosos para Bogotá

3) El programa de carreteras departamentales y el proyecto de ampliación y mejoramiento de la carretera Bogotá-Buenaventura

4) EAE de ciudades grandes e intermedias, a partir de la sistematización de los planes de ordenamiento territorial

5) Evaluación ambiental del Plan de Expansión Sectorial en Generación y Transmisión de Energía

6) Diseño de una estrategia integral de fortalecimiento institucional para la gestión ambiental en el sector transporte y de sus subsectores. Hasta aquí se trata de iniciativas e intenciones aisladas de poner en práctica las EAEs.

En Colombia se da estatus legal a las EAE con la promulgación del actual Plan Nacional de Desarrollo 2002-2006, cuando dentro de sus programas de Sostenibilidad Ambiental de la Producción Nacional (art. 8, literal B, numeral 8) se establece la "realización de evaluaciones ambientales estratégicas para sectores productivos críticos “(...) de igual forma, pero indirectamente, a través de la Ley Orgánica de Planeación (Ley 152 de 1994), en cuanto a que se comparten las orientaciones de la EAE en el sentido de que, en el plan de desarrollo, se deben incorporar estrategias, programas y proyectos que estimen los costos y beneficios ambientales. Hasta el momento, las iniciativas de EAE - incluyendo la del PND- han sido orientadas hacia los sectores de desarrollo y productivos, es decir a PPP, pero ninguno que apunte o decisiones de político relacionadas con la planificación, protección, aprovechamiento y manejo integral de los recursos naturales. Sin embargo, es de esperarse, al menos en teoría, que a futuro se desarrollen EAE para sectores como el Minero-Energético, el de Transporte, el Agropecuario (incluyendo el aprovechamiento forestal de bosques naturales), el Saneamiento Básico (incluyendo el ordenamiento de recursos hídricos superficial y subterráneo) y Turismo, y para los Ámbitos Nacional y Territorial, a través de los Planes de Desarrollo del nivel nacional, departamental y municipal y los Planes de Ordenamiento Territorial. Lo anterior, teniendo en cuenta los resultados de "Una propuesta conceptual y metodológica para la Aplicación de EAE en Colombia" elaborada por el Departamento Nacional de Planeación (DNP) en el año 2004. Finalmente, se debe destacar que la respuesta de Colombia a lo adopción de EAE en los procesos de decisión PPP como lo fuera en su momento la adopción de los EIA, tiene de alguno forma su origen en compromisos bilaterales adquiridos (Gilberto Vallejo Moreno). 
Revista Geográfica Digital. IGUNNE. Facultad de Humanidades. UNNE. Año 14. № 28.

Julio - Diciembre 2017. ISSN 1668-5180 Resistencia, Chaco

\section{RESULTADOS}

\section{¿Cómo aplicar la EAE en proyectos de infraestructura?}

Los beneficios más comúnmente percibidos de la EAE, se describen como: la posibilidad de una consideración amplia de efectos sobre el ambiente y alternativas a considerarse; la anticipación de participación pública en discusiones y el incremento de la efectividad en decisiones relacionadas al desarrollo de proyectos con ahorros en costo y dinero, así como el consecuente fortalecimiento de los procesos de decisión estratégica (Dutra de Oliveira, Montaño and Souza, 2013).

Por lo tanto, a continuación se discutirá acerca de los beneficios potenciales del uso de la EAE para promover el estatus de los niveles estratégicos de decisión en los planes y programas de infraestructura de transporte. Mediante un caso de estudio aplicado en el estado de Sao Paulo, el artículo aboga al uso sistemático de la EAE en orden de reforzar las valoraciones de impacto en el país.

No obstante, para que la práctica de la EAE sea efectiva, debe estar basada en la evidencia y en el cumplimiento de los objetivos; cumplimiento de la línea base; flexible y adaptable a contextos específicos. Se reconoce que la efectividad de la EAE se perfecciona con la práctica sistemática, como una consecuencia del aprendizaje organizacional. (Dutra de Oliveira, Montaño and Souza, 2013).

Aunque algunos tienen un sistema formal, la EAE sigue siendo aplicada frecuentemente sin requerimientos legales que especifiquen objetivos y procedimientos a seguir bajando niveles estratégicos de decisión.

La habilidad de identificar efectos significativos al ambiente causados por las acciones estratégicas y las interacciones con otros niveles, es un aspecto clave del marco metodológico de la EAE.

Según la guía de descripción y aplicación de la Evaluación Ambiental Estratégica elaborada por el Grupo del Banco Mundial, hay dos enfoques para una EAE efectiva: el primer enfoque es con centro en el impacto y el segundo con centro en las instituciones, pero debemos sumar a ello un enfoque combinado de la EAE; en la siguiente tabla se describe cada uno de los enfoques mencionados.

\begin{tabular}{|c|c|c|}
\hline \multicolumn{3}{|c|}{ Enfoques de la Evaluación Ambiental Estratégica } \\
\hline Con centro en el Impacto & $\begin{array}{l}\text { Con centro en las } \\
\text { instituciones }\end{array}$ & Enfoque combinado \\
\hline $\begin{array}{l}\text { Éste se enfoca en los impactos } \\
\text { materiales y antrópicos de un } \\
\text { plan de desarrollo propuesto. } \\
\text { Los impactos materiales o } \\
\text { físicos, incluyen proyectos de } \\
\text { infraestructura y cambios en el } \\
\text { uso del suelo. Los impactos } \\
\text { antrópicos incluyen la } \\
\text { promoción de crecimiento } \\
\text { económico o de la salud y la } \\
\text { seguridad. Para este enfoque, } \\
\text { el criterio de toma de } \\
\text { decisiones se centra en el } \\
\text { sistema de incentivos y en las } \\
\text { instituciones apropiadas para }\end{array}$ & 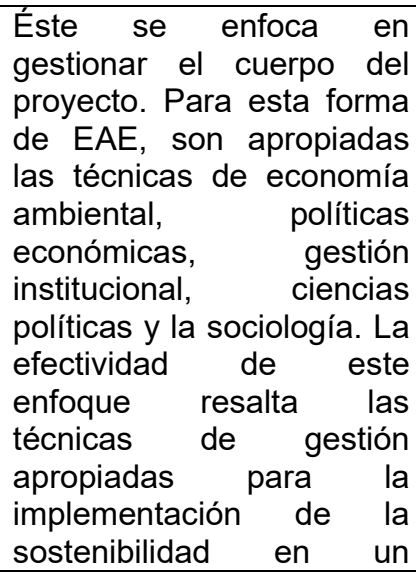 & $\begin{array}{l}\text { Una amplia EAE incluirá } \\
\text { estrategias de los dos } \\
\text { enfoques anteriores. Las } \\
\text { condiciones del proyecto } \\
\text { determinarán cual metodología } \\
\text { es la más pertinente. Por } \\
\text { ejemplo, una EAE de una } \\
\text { central hidroeléctrica será } \\
\text { desarrollada usando un } \\
\text { enfoque con centro en el } \\
\text { impacto. Esta se centrará en } \\
\text { los impactos ambientales de la } \\
\text { construcción y la operación } \\
\text { propuesta para el proyecto } \\
\text { hidroeléctrico y en el impacto }\end{array}$ \\
\hline
\end{tabular}

Publicado en formato digital: Prof Sandra Milena Carabuena Mendoza LA EVALUACIÓN AMBIENTAL ESTRATÉGICA COMO INSTRUMENTO DE VALORACIÓN ESPACIAL. ANÁLISIS DE SU IMPLEMENTACIÓN EN PROYECTOS DE INFRAESTRUCTURA VIAL. Revista Geográfica Digital. IGUNNE. Facultad de Humanidades. UNNE. Año 14. № 28. Julio Diciembre 2017. ISSN 1668-5180. Resistencia, Chaco. En: http://hum.unne.edu.ar/revistas/geoweb/default.htm 
Revista Geográfica Digital. IGUNNE. Facultad de Humanidades. UNNE. Año 14. № 28.

Julio - Diciembre 2017. ISSN 1668-5180 Resistencia, Chaco

\begin{tabular}{|l|l|l|}
\hline atraer la inversión, fomentar el & proyecto de desarrollo. & de la economía de la \\
ahorro o el consumo y & producción de la electricidad. \\
promover las exportaciones. & & \\
Un componente crítico de una & & \\
evaluación ambiental es & & \\
analizar adecuadamente el & & \\
ambiente para gestionar & & \\
óptimamente el crecimiento a & & \\
una taza sostenible. &
\end{tabular}

Tabla 2. Enfoques de la Evaluación Ambiental Estratégica

A continuación se mencionan algunos países que han trabajado la $E A E$, a nivel de proyectos:

\begin{tabular}{|c|c|c|}
\hline PAís & TEMA & SÍNTESIS \\
\hline BRASIL & $\begin{array}{l}\text { Proyectos de } \\
\text { infraestructura }\end{array}$ & $\begin{array}{l}\text { Brasil ha estado trabajando básicamente } \\
\text { con la EAE adaptando procedimientos de } \\
\text { proyectos EIA. Notablemente, EAE ha } \\
\text { sido aplicado en Brasil para proyectos } \\
\text { estructurales, amplios proyectos de } \\
\text { infraestructura que pueden cambiar } \\
\text { significativamente el desarrollo de una } \\
\text { región }\end{array}$ \\
\hline MÉXICO & $\begin{array}{l}\text { Reforma del } \\
\text { turismo en México }\end{array}$ & $\begin{array}{l}\text { Políticas para el desarrollo sostenible del } \\
\text { turismo y el desarrollo de la campaña } \\
\text { "México limpio y querido" mejoró la } \\
\text { recopilación y difusión de los datos } \\
\text { relacionados con la demanda y oferta de } \\
\text { turismo existente para facilitar la } \\
\text { planeación del turismo sostenible; } \\
\text { desarrollar y actualizar los planes de uso } \\
\text { del suelo; fortalecer el marco institucional } \\
\text { que promueve la interacción entre todas } \\
\text { las partes interesadas; desarrollar los } \\
\text { incentivos fiscales para promover la } \\
\text { innovación y la sostenibilidad ambiental. }\end{array}$ \\
\hline COLOMBIA & $\begin{array}{c}\text { Reforma ambiental } \\
\text { en Colombia }\end{array}$ & $\begin{array}{l}\text { Elaboración del programa de préstamo } \\
\text { para política de desarrollo y el proyecto } \\
\text { de asistencia técnica para (a) mejorar la } \\
\text { efectividad y la eficacia del sistema } \\
\text { nacional ambiental de Colombia y (b) } \\
\text { integrar los principios de desarrollo } \\
\text { sostenible dentro de los sectores políticos } \\
\text { clave, protegiendo los grupos de debate } \\
\text { más vulnerables que llevaron a la } \\
\text { aprobación de la ley de control de la } \\
\text { contaminación atmosférica. } \\
\text { información base a medida que el } \\
\text { gobierno fortaleció la gobernabilidad de } \\
\text { las autoridades ambientales regionales y } \\
\text { la conexión entre las prioridades locales y } \\
\text { nacionales. }\end{array}$ \\
\hline
\end{tabular}

Tabla 3. Países que están manejando la Evaluación Ambiental Estratégica 
Revista Geográfica Digital. IGUNNE. Facultad de Humanidades. UNNE. Año 14. № 28.

Julio - Diciembre 2017. ISSN 1668-5180 Resistencia, Chaco

\section{* Formas de implementación de la EAE}

Idealmente, este proceso debe comenzar en una fase temprana de la formulación del proyecto extenderse hasta la fase de implementación y terminar solo después de que se haya realizado un monitoreo completo de todo el proyecto. Con base en el marco procedimental de una EAE corriendo en paralelo con la elaboración del plan de internacionalización puede ser resumido en los siguientes ítems: Guidance Notes on Tools for Pollution Management.

- Debe estabilizar los criterios generales que guíen el estudio de alternativas en la región de Ribeirao Preto como un todo.

- Crea una oportunidad de incluir aspectos ambientales en escenarios tempranos de decisión estratégica, así como colaboraciones con los niveles de decisión;

- Podrá incluir la visión a largo plazo de las políticas de transporte del estado;

- Habrá más espacio para buscar alternativas compatibles y soluciones que encajen con el umbral ambiental en primer lugar y para cada nivel estratégico de decisión.

- Debe promover la conciliación de los niveles de decisión entre el estado y las estrategias regionales y locales;

- Debe incluir acciones proactivas enfocadas en el mejoramiento de los impactos positivos de los diferentes niveles de decisión;

- Permite la evaluación sostenible de alternativas que no están restringidas a las especificaciones de los proyectos.

Para que la implementación de la EAE sea eficaz, se debe ajustar al contexto y condiciones del proyecto, para ello, se debe incorporar los impactos ambientales acumulativos de varios proyectos simultáneos propuestos por un plan de desarrollo.

\section{Esquema detallado del procedimiento de la EAE.}

\begin{tabular}{|c|c|}
\hline Fase & Procedimiento \\
\hline $\begin{array}{l}\text { Fase de identificación } \\
\text { (Screening) }\end{array}$ & $\begin{array}{c}\text { Identificar si la propuesta de } \\
\text { plan o programa es } \\
\text { susceptible de someterse a } \\
\text { EAE }\end{array}$ \\
\hline Fase de inicio & Documentación inicial \\
\hline Fase de alcance o scoping & $\begin{array}{c}\text { Determinar el alcance de la } \\
\text { evaluación (Consultas } \\
\text { previas a la autoridad } \\
\text { ambiental) }\end{array}$ \\
\hline $\begin{array}{l}\text { Fase de Elaboración del } \\
\text { ISA (Informe de } \\
\text { sostenibilidad Ambiental). }\end{array}$ & $\begin{array}{c}\text { Evaluar los posibles efectos } \\
\text { en el medio ambiente de la } \\
\text { propuesta, comparando su } \\
\text { contenido con los objetivos } \\
\text { medioambientales y buscar } \\
\text { alternativas. }\end{array}$ \\
\hline \multirow[t]{2}{*}{$\begin{array}{l}\text { Fase de elaboración de la } \\
\text { memoria ambiental }\end{array}$} & $\begin{array}{c}\text { Tener en cuenta los } \\
\text { resultados de fases } \\
\text { anteriores y de las consultas } \\
\text { realizadas a la hora de } \\
\text { adoptar el plan o programa. }\end{array}$ \\
\hline & $\begin{array}{c}\text { Elaboración conjunta de la } \\
\text { memoria ambiental }\end{array}$ \\
\hline $\begin{array}{l}\text { Fase de consideración del } \\
\text { ISA y de las consultas }\end{array}$ & $\begin{array}{l}\text { Integración del ISA y de la } \\
\text { MAC en el PP. }\end{array}$ \\
\hline
\end{tabular}

Tabla 4. Procedimiento de la EAE - Guía de evaluación ambiental estratégica en Colombia

Publicado en formato digital: Prof. Sandra Milena Carabuena Mendoza. LA EVALUACION AMBIENTALESTRATÉGICA (E COMO INSTRUMENTO DE VALORACIÓN ESPACIAL. ANÁLISIS DE SU IMPLEMENTACIÓN EN PROYECTOS DE INFRAESTRUCTURA VIAL. Revista Geográfica Digital. IGUNNE. Facultad de Humanidades. UNNE. Año 14. № 28. Julio Diciembre 2017. ISSN 1668-5180. Resistencia, Chaco. En: http://hum.unne.edu.ar/revistas/geoweb/default.htm 
Revista Geográfica Digital. IGUNNE. Facultad de Humanidades. UNNE. Año 14. № 28.

Julio - Diciembre 2017. ISSN 1668-5180 Resistencia, Chaco

\section{CONCLUSIONES}

$\checkmark$ La Evaluación Ambiental Estratégica debe adoptar objetivos claros y bien definidos, incluyendo competencias formales y procedimientos responsables para llenar las deficiencias de los proyectos de las Evaluaciones de Impacto Ambiental.

$\checkmark$ El uso sistemático de la EAE, puede contribuir al aprendizaje social e institucional promoviendo niveles de decisión.

$\checkmark$ El concepto de la EAE aún no se unificado pero es entendido como un proceso sistemático que incorpora las consideraciones y criterios ambientales en los momentos claves del proceso de planeación de decisiones estratégicas.

$\checkmark$ El desarrollo de una EAE debe de alguna manera garantizar el equilibrio entre la sostenibilidad ambiental y los planes de desarrollo socioeconómico de un país, un sector y/o región".

$\checkmark$ Para implementar la EAE se debe comenzar en una fase temprana de la formulación del proyecto y extenderse hasta la fase de implementación y terminar solo después de que se haya realizado un monitoreo completo de todo el proyecto. 
Revista Geográfica Digital. IGUNNE. Facultad de Humanidades. UNNE. Año 14. № 28.

Julio - Diciembre 2017. ISSN 1668-5180 Resistencia, Chaco

\section{BIBLIOGRAFÍA}

- Agencia Portuguesa Do Ambiente. (2012) Guía de Mejores Prácticas para la Evaluación Ambiental Estratégica: Orientaciones metodológicas para un pensamiento estratégico en EAE. Recuperado de:

www.goredelosrios.cl/cultura2/wp-content/uploads/2016/02/Guía de-Mejores-Prácticas-para-laEvaluación-Ambiental-Estratégica-Maria-do-Rosário Partidário.pdf.

- Castillo Arévalo L. (2013). Análisis de la Evaluación Ambiental Estratégica y su articulación en el proceso de consulta previa En Colombia. (Tesis de maestría). Pontificia Universidad Javeriana. Bogotá D.C., Colombia.

- Conferencia Europea de Ministros de Transporte - ECMT. (1998). Evaluación Ambiental Estratégica en el Sector del Transporte. Recuperado de: http://www.oecd.org/cem/.

- Gómez Jorge. (Diciembre 2010). La Evaluación Ambiental Estratégica (EAE) en Colombia: Su adopción, criterios para la estructuración de su procedimiento administrativo y su aplicación a los planes de ordenamiento territorial. Revista científica Universidad Distrital. Revista No 12. Páginas $48-62$.

- Guidance Notes on Tools for Pollution Management. Grupo del Banco Mundial: James Warren Evans / Mary Barton-Dock (Directores, Departamento de Medio Ambiente, Banco Mundial), Bilal Rahill (Gerente Senior, IFC) y Frank Lysy / Ravi Vish (Director, MIGA).

- Lee, N. y Walsh, F. (1992). Strategic Environmental Assessment: An Overview. Project Appraisal 7: paginas 126-36. Recuperado de: http://www.tandfonline.com/doi/pdf/10.1080/02688867.1992.9726853

- Ministerio de Ambiente, Vivienda y Desarrollo Territorial. (2008). Guía de Evaluación Ambiental Estratégica. Recuperado de:

- http://www.minambiente.gov.co/images/AsuntosambientalesySectorialyUrbana/pdf/Evaluaci\%C3 \%B3n Ambiental Estrategica/Guia de evaluci\%C3\%B3n ambiental Estrat\%C3\%A9gica.

- Strategic Environmental Assessment to Improve Infrastructure Impact Assessments in Brazil. Isabel Silva Dutra de Oliveira, Marcelo Montaño, Marcelo Pereira de Souza. 2013. 